\title{
Editorial November 2014
}

DOI 10.1515/epoly-2014-0190

In the September editorial of e-Polymers we addressed the importance of relevant research in polymer science. It was also pointed out that relevance could be a pointof-view. In the present issue of e-Polymers, polymers with high biorelevance are reported. Biorelevant polymers are a particular point-of view of relevant polymer research. The field of biorelevant polymers itself covers different aspects which are nicely reflected by the different contributions in this issue of e-Polymers. One important aspect is the use of nature-based molecules or in other words, renewable materials, for the synthesis of monomers and polymers or the modification of nature-based polymers by man-made molecules. Boonyarattanakalin et al. report in their contribution on the mannopyranan polymers based on ringopening polymerization of mannose derivatives. These polymers were obtained in considerable molecular weight but what makes these polymers particularly attractive is the control on the stereochemistry on a polymer level. Another approach to biobased polymers was undertaken by Zammanloo and coauthors using derivatized L-aspartic acid which yielded co-poly(amideimides) with aromatic moieties. These copolymers were mostly amorphous and thereby transparent which makes them highly attractive for optical applications in combination with their optical activity and high thermal stability. Torres et al. used polyhydroxybutyrate as a highly biorelevant polymer, which is a well-known biodegradable polymer and modified it by radiation-induced grafting with oilgo(vinylalcohol). Oil-in-water emulsification resulted upon extraction relatively small particles which makes them highly attractive for pharmaceutical applications. Saif et al. address in their contribution, biorelevant polymers in the important field of antimicrobial polymers which are gaining, as potential non-leaching anti-microbial materials, more and more importance over classical antimicrobial materials like silver nanoparticles. Saif et al. show that the antimicrobial activity can be tuned directly by the quantity of the antimicrobial benzimidazolium moiety in the copolymer. A somewhat surprising but highly interesting approach to gain biorelevance was applied by Bhullar who show that the auxetic polyurethanes foams, which expand upon stretching, could be of use for biomedical implants.

As in previous editorials of e-Polymers we introduce the members of our distinguished editorial board, this time it is Professor Stephen Eichhorn from University of Exeter, UK. Prof. Eichhorn holds a full professorship for Materials Science and is very well-known for his excellent research on materials from renewable resources. His particular research focus is on nanocellulose and biomimetic materials. In combination with his successful research in electrospinning and his in-depth interest in Raman spectroscopy and other characterization techniques he has an ideal platform for ground breaking research. He also utilizes this expertise for the benefit of $e$-Polymers in his ongoing effort to disseminate the high level of scientific progress which is the core-task of e-Polymers.

Andreas Greiner and Seema Agarwal 